\title{
Efeitos da cafeína na performance de exercícios de endurance
}

\author{
Effects of caffeine on performance of endurance exercises
}

Giuliano Roberto da Silva, ${ }^{1}$ Marcelo Rodrigo Tavares, ${ }^{1}$ Gerusa Dias Siqueira Vilela Terra, ${ }^{1}$ Cassiano Merussi Neiva, ' César Augusto Costa Rodrigues, 'Yvan Fernandes Vilas Boas,' Tatiana de Araújo Barcelos 'Universidade José do Rosário Vellano (UNIFENAS), Alfenas, MG, Brasil.

Recebido em: Setembro 2015 / Aceito em: Dezembro 2015 giumusc@gmail.com

\section{RESUMO}

Objetivo: verificar os efeitos da cafeína na performance de exercícios de endurance, através do teste de exaustão na bicicleta ergométrica. Método: participaram 50 indivíduos do sexo masculino, submetidos ao teste de exaustão em cicloergômetro após ingestão de $6 \mathrm{mg} / \mathrm{kg}$ de cafeína (CAF) ( $\mathrm{n}=25$; grupo cafeína), e $6 \mathrm{mg} /$ $\mathrm{kg}$ de placebo (PLA) ( $\mathrm{n}=25$; grupo placebo), 40 minutos antes do teste. Os dados foram tratados estatisticamente, submetidos ao teste $t$ de Student $(p<0,05)$. Resultados: os resultados mostraram: aquecimento: grupo CAF apresentou frequência cardíaca ( $F C$ ) média maior (89 bpm) quando comparada ao grupo PLA (85 bpm) $(p=0,005)$; teste: os grupos CAF e PLA foram iguais em relação à $\mathrm{FC}(\mathrm{CAF}=134 \mathrm{bpm} ; \mathrm{PLA}=134 \mathrm{bpm})$; não houve diferença significativa nos tempos de exaustão entre os grupos $(p=0,165)$. Considerações finais: na dosagem de $6 \mathrm{mg} / \mathrm{kg}, 40$ minutos antes do exercício, a cafeína não parece exercer efeitos na performance de exercícios de endurance.

Palavras-chave: Fadiga; Cafeína; Exercício de endurance.

\section{ABSTRACT}

Objective: to investigate the effects of caffeine on performance of endurance exercises, through the exhaustion test on the exercise bike. Method: participants were 50 males, submitted to exhaustion test on a cycle ergometer after intake of $6 \mathrm{mg} / \mathrm{kg}$ of caffeine (CAF) ( $n=25$; caffeine group), and $6 \mathrm{mg} / \mathrm{kg}$ for placebo (PLA) ( $n=25$; placebo group) 40 minutes before the test. The data were treated statistically, submitted to the Student's t test $(p<0.05)$. Results: the results showed: Heating: CAF group had heart rate (HR) highest average (89 bpm) when compared to the PLA group (85 bpm) ( $p=0.005) ;$ test: the CAF and PLA groups were equal with respect to the $F C(F C=134 \mathrm{bpm}$; $P L A=134 \mathrm{bpm})$; there was no significant difference in exhaustion times between the groups ( $p=0.1658)$. Closing remarks: the dose of $6 \mathrm{mg} / \mathrm{kg}, 40$ minutes before exercise, caffeine does not seem to have effects on the performance of endurance exercises.

Keywords: Fatigue; Caffeine; Endurance exercise.

\section{INTRODUÇÃO}

A fadiga tem sido apontada como fator limitante do desempenho atlético e sua origem depende da duração do exercício, intensidade, tipo de fibras que estão sendo recrutadas na ação muscular, ${ }^{1}$ que são (I, Ila, Ilb, liab), ${ }^{2}$ e do grau de aptidão individual, ${ }^{3}$ podendo ocorrer em locais diferenciados e também no decorrer do trajeto entre o sistema nervoso central e a musculatura contráctil utilizada, e portanto, pode ser dividida em central e periférica. ${ }^{4} \mathrm{~A}$ fadiga pode ser desencadeada por várias situações, sendo: suprimento energético; acoplamento excitação-contração; via excitatória para motoneurômios; excitabilidade dos motoneurônios; aferência excitatória para centros motores superiores; transmissão neuro-muscular; excitabilidade do sarcolema e acúmulo de metabólitos. ${ }^{5}$

Fadiga central, é ocasionada quando cognições e ações que necessitam de um aumento de performance ou esforço; é interrompida sem evidências de redução dos fatores motores periféricos. ${ }^{6}$ O sistema nervoso central acomete a fadiga se houver uma redução da frequência de disparos e quantidade de unidades motoras excitadas. ${ }^{5}$ Fadiga periférica diz respeito a falhas do sistema neuromuscular na periferia, em que eventos mecânicos, neurais e energéticos comprometeriam a 
transmissão nervosa periférica ou o processo de contração muscular. ${ }^{7}$

Diversas substâncias, legais ou não, têm sido utilizadas, previamente à realização de exercícios de natureza aeróbia e anaeróbia, com o intuito de protelar a instalação do processo de fadiga e, consequentemente, aprimorar a performance atlética, e a cafeína é uma delas. Esta foi removida da lista de substâncias proibidas pela agência mundial anti-doping (World Anti-Doping Agency - WADA), e é encontrada em inúmeros alimentos e bebidas, sendo de fácil acesso, justificando seu alto índice de consumo entre adultos por todo mundo. É um ergogênico que desperta grande interesse no meio científico, possuindo uma estreita relação com o meio esportivo. ${ }^{8}$

A melhora do desempenho atlético se dá de acordo com estudos que investigaram o impacto da ingestão de cafeína em esportes de endurance como: corridas, ciclismo, natação e remo. ${ }^{9}$ Todavia, há uma carência de estudos que analisaram os efeitos da cafeína em esportes intermitentes, entretanto, mesmo com essas limitações, observam-se evidências de que a cafeína também melhora a performance neste tipo de esportes, que em tese, se dá através da mobilização de ácidos graxos livres do tecido adiposo, aumentando o suprimento de gordura ao músculo, o que gera economia no uso do glicogênio e melhora da função neuromuscular; esse fato prolonga o tempo de exercício, ou seja, protela a fadiga..$^{10}$ Diante do exposto, o objetivo deste estudo foi verificar os efeitos da cafeína no comportamento/ gesto motor em exercícios de endurance, através do teste de exaustão na bicicleta ergométrica em um teste incremental máximo.

\section{MÉTODO}

A pesquisa foi realizada após aprovação do Comitê de Ética em Pesquisa da Universidade José do Rosário Vellano (UNIFENAS), com parecer $n^{\circ}$ 776.656. Participaram do estudo 50 indivíduos do sexo masculino, hígidos com boa saúde, que não tinham prática específica com este tipo de teste ergométrico. Foram convidados a participar voluntariamente, mediante assinatura do Termo de Consentimento Livre e Esclarecido (TCLE). Estas e demais considerações éticas se basearam na resolução 466/12 do Conselho Nacional de Saúde (CNS).

Os participantes foram universitários dos cursos de Educação Física, Fisioterapia, Odontologia e Biomedicina, com idade média de 21,5 anos $( \pm 1,9)$, IMC de $23,1 \mathrm{Kg} / \mathrm{m}^{2}( \pm 0,83)$, altura média de $1,7 \mathrm{~m}$, pouco ativos fisicamente (indivíduos que não tinham uma regularidade semanal de exercícios físicos sitemáticos), sem controle alimentar (por um nutricionista e/ou nutrólogo), habitação (repúblicas estudantis em sua maioria; 95\% destes) e não tinham nenhuma restrição em relação a ingestão de cafeína. Foram instruídos a chegar no local da prática do teste descansado, hidratado e ainda não terem realizado nenhum tipo de exercício de membro inferior em até 72 horas antes do teste. Os sujeitos foram subdividos aleatoriamente em dois grupos equalitários e instruídos a ingerirem $6 \mathrm{mg} / \mathrm{kg}$ de peso de cafeína (grupo CAF) ou placebo (açúcar- grupo PLA), 40 minutos antes do teste. Os avaliadores do estudo sabiam do conteúdo das substâncias ingeridas pelos universitários, porém estes não sabiam qual substância que cada um iria ingerir. Tudo isso aconteceu em comum acordo entre a equipe avaliadora do laboratório de Performance Humana da Universidade e os avaliados.

Todos os testes foram realizados ao mesmo tempo do dia ( \pm 14 horas). Os 50 sujeitos foram submetidos ao teste no cicloergômetro Maxx ${ }^{\circledR}$ (Hidrofit, Brasil) padrão Monark ${ }^{\circledR}$, sendo monitorada a frequência cardíaca através do frequencímetro Oregon Scientific ${ }^{\circledR}$ SE 102L.

$\mathrm{O}$ teste ergométrico constou das seguintes etapas: I) Adequação dos sujeitos à bicicleta; II) Alongamento de membros inferiores; III) Aquecimento (7 minutos na bicicleta a ser utilizada, sem carga, girando a partir de $40 \mathrm{rpm}$ até evoluir para $70 \mathrm{rpm}$ ); IV) Teste: os sujeitos permaneceram na bicicleta ergométrica, sentados, girando a pedivela a uma frequência mínima de $70 \mathrm{rpm}$ e máxima de $90 \mathrm{rpm}$. A atividade iniciou sem carga, sendo acrescida uma carga de $0,5 \mathrm{~kg}$ a cada 2 minutos na resistência da bicicleta. O teste chegou ao fim quando o sujeito não conseguiu manter os $70 \mathrm{rpm}$ ou pedia para parar; V) Desaquecimento: após o término do teste, foram retirados os pesos e os participantes fizeram um desaquecimento de 2 minutos na bicicleta sem carga, girando de $30 \mathrm{rpm}$ a $45 \mathrm{rpm}$.

O teste estatístico utilizado para verificar a existência de diferença significativa entre os grupos em comparação foi o teste $\mathrm{t}$ de Student para diferenças entre as médias ao nível de $5 \%$ de probabilidade. 0 software estatístico utilizado para a realização das análises foi o Sisvar ${ }^{\circledR}$.

\section{RESULTADOS}

A Figura 01 demonstra a média de frequência cardíaca no período de aquecimento (1 a 7 minutos). Observou-se uma diferença significativa entre os grupos cafeína e placebo $(p=0,005)$. O grupo cafeína apresentou uma frequência cardíaca média maior $(89$ bpm), no período de 1 a 7 minutos, quando comparado à frequência cardíaca média do grupo placebo (85 bpm). No período do teste propriamente dito (tempo superior a 7 minutos, até a exaustão), os dados de frequência cardíaca encontrados não apresentaram diferença significativa entre os grupos CAF e PLA $(p=0,951)$.

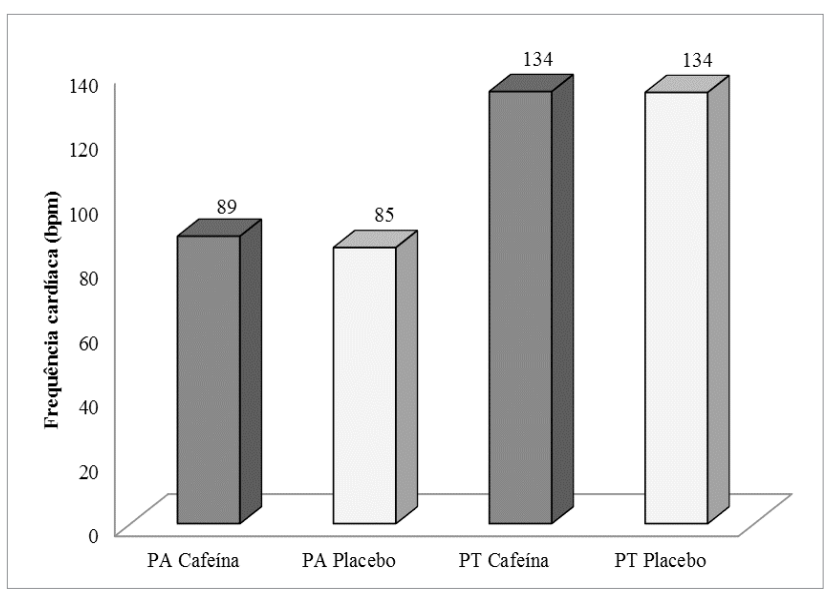

*bpm: batimentos por minuto; PA: período de aquecimento; PT: período de teste.

Figura 1 - Frequência Cardíaca Média (FCM) no PA e PT. 
Em relação aos tempos de exaustão, os resultados revelaram que não houve diferença significativa entre os tempos de exaustão para o grupo de indivíduos que ingeriu cafeína quando comparado ao grupo de indivíduos que não ingeriu cafeína $(p=0,165)$, conforme observado na Figura 02.

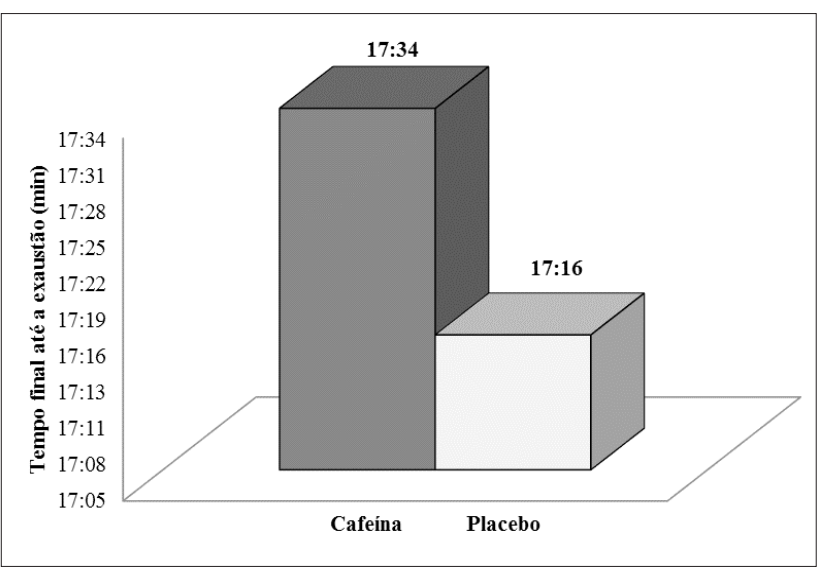

Figura 2 - Tempo final até a exaustão.

\section{DISCUSSÃO}

O presente estudo demonstrou que a cafeína, na dosagem de $6 \mathrm{mg} / \mathrm{kg}$, 40 minutos antes do teste exaustão em cicloergômetro, não foi eficiente na melhora da performance dos indivíduos. Da mesma forma, estudo não observou mudanças significativas na performance de atletas em protocolo de sprints repetidos, realizados após ingestão de $6 \mathrm{mg} / \mathrm{Kg}$ de cafeína. ${ }^{11}$ Fato semelhante foi encontrado em um estudo prospectivo, randomizado, realizado com 43 indivíduos, submetidos à suplementação de cafeína e a uma mistura de outros suplementos (creatina, betaína e um extrato de dendrobium), durante 06 semanas. Os resultados demonstraram que não houve melhorias no desempenho e na composição corporal; contudo, houve melhoria significativa na sensação subjetiva de esforço $(p<0,024)$ e na concentração $(p<0,041)$, gerando níveis mais elevados de foco no exercício. ${ }^{12}$

Todavia, pesquisa ${ }^{13}$ utilizou a mesma quantidade de cafeína do estudo anterior em atletas de esportes coletivos, os quais verificaram melhora na capacidade de realização de sprints repetidos, que de acordo com Spencer e colaboradores, ${ }^{14}$ é uma boa indicativa no desempenho em modalidades coletivas. Estes fatos relacionados à melhora da motivação e diminuição da sensação subjetiva de esforço também foram relatados em outro estudo. ${ }^{15}$ Os autores afirmam que a cafeína, ingerida em doses moderadas $(3-6 \mathrm{mg} / \mathrm{kg}$ de massa corporal), tem um efeito sobre o sistema nervoso central, através do antagonismo dos receptores de adenosina, sugerindo influência nos mecanismos dopaminérgicos, possíveis fatores para explicar como os componentes de motivação e os processos cerebrais de alta ordem estão envolvidos no controle motor. Segundo os autores, a cafeína mantém uma concentração de dopamina superior especialmente nas áreas do cérebro associadas à "atenção". Através desta interação neuroquímica, a cafeína melhoraria a atenção sustentada, vigilância e reduziria os sintomas de fadiga. Esses fatos foram reforçados em outro estudo que analisou mecanismos bioquímicos consistentes, envolvendo proteínas como DARPP-32 (dopamina e fosfoproteína regulada pelo AMPc). ${ }^{16}$

Foi relatado em um estudo que em modelos tradicionais, cujo foco é examinar a potência, foi encontrado um efeito mínimo da cafeína como ergogênico e que, em metodologias mais específicas, com menor duração e maior explosão, como em exercícios de alta intensidade e intermitentes, há uma melhor resposta da cafeína no desempenho atlético. ${ }^{17}$

Contrapondo-se a esses resultados, em uma pesquisa com ciclistas treinados, submetidos a duas sessões experimentais, com ingestão aleatória de cafeína na mesma dosagem utilizada no presente estudo, uma hora antes do teste, não houve melhora no desempenho anaeróbio intermitente. Além disso, demonstrou redução na potência média nos indivíduos sob uso de cafeína, podendo sugerir ser desvantajoso seu uso durante esforços máximos intermitentes. ${ }^{18}$

Em uma investigação sobre a relação entre a cafeína e a potência crítica em bicicleta ergométrica, os resultados encontrados corroboram com os do presente estudo. Nesta pesquisa, também de caráter duplo-cego, foram administradas dosagens de $6 \mathrm{mg} / \mathrm{kg}$ de cafeína pura para um grupo e maltodextrina para outro grupo. Os indivíduos foram submetidos a quatro testes de carga constante em cicloergômetro em intensidades de $80,90,100$ e $110 \%$ de potência máxima, até a exaustão máxima, para se determinar a potência crítica. Conclui-se que a ingestão de cafeína não melhorou o desempenho da potência crítica. ${ }^{19}$

Já, em uma investigação sobre os efeitos metabólicos e de desempenho da ingestão da cafeína em comparação ao consumo de café durante o exercício de resistência, chegou-se à conclusão que, tanto a cafeína $(5 \mathrm{mg} / \mathrm{kg})$, quanto o café $(5 \mathrm{mg} / \mathrm{kg})$ consumidos uma hora antes do exercício podem melhorar o desempenho em exercícios de resistência. ${ }^{20}$

Entretanto, sugerem-se pesquisas futuras, para melhores investigações, e que esta seja adaptada ao nível técnico, condição de somatotipo e nível nutricional com a presença de cafeína, aproximando mais a realidade da população investigada.

\section{CONSIDERACÕ̃ES FINAIS}

Quando ingerida em dosagens de aproximadamente $6 \mathrm{mg} / \mathrm{kg}, 40$ minutos antes do exercício, a cafeína não parece exercer efeitos na performance de endurance. Contudo, devido às diversas controversas encontradas na literatura, recomenda-se que mais pesquisas sejam realizadas sobre este tema, determinando seu real efeito nas diferentes formas de exercícios físicos.

\section{REFERÊNCIAS}

1. Powers SK, Howley ET. Fisiologia do exercício. Barueri: Manole, 2006; $116 \mathrm{p}$

2. Pereira B, Souza Júnior TP. Metabolismo Celular e Exercício Físico. São Paulo: Phorte, 2004; 145 p. 
3. Fitts RH. Cellular, molecular, and metabolic basis of muscle fatigue. In: FITTS RH. Handbook of physiology. Oxford: Oxford University Press; 1996. p. 1150-83.

4. Giannesinii B, Cozzone PJ, Bendahan D. Non invasive investigation of muscular fatigue: Metabolic and electromyografic components. Biochimie (Paris) 2003;85(9): 873-883. doi: 10.1016/S0300-9084(03)00124-X

5. Gibson H, Edwards RHT. Muscular exercise and fatigue. Sports Medicine 1985;2(2):120-32. doi: 10.2165/00007256198502020-00004

6. Morgan RM, Parry AM, Arida RM, Matthews PM, Davies B, Castell LM. Effects of elevated plasma tryptophan on brain activation associated with the Stroop task. Psychopharmacology 2007;190(3):383-389. doi: 10.1007/ s00213-006-0609-7

7. Westerblad H. Cellular mechanisms of fatigue in skeletal muscle. American J Physiol 1991;261(2):195-209. doi: 10.1007/978-1-4419-9029-7_50

8. Caputo F, Aguiar RA, Turnes T, Silveira BH. Cafeína e desempenho anaeróbio. Rev Bras Cineantropom Desempenho Hum 2012;14(2):602-614. doi: 10.5007/1980-0037.2012v1 4n5p602

9. Pereira A, Cyrino ES, Avelar A, Segantin AQ, Altimari JM, Trindade MCC, Altimari LR. A ingestão de cafeína não melhora o desempenhodeatletas dejudô. Motriz, RioClaro2010;16(3):714722. doi: 10.5016/1980-6574.2010v16n3p714

10. Doherty M, Smith P, Hughes M, Davison R. Caffeine lowers perceptual response and increases power output during highintensity cycling. Journal of Sports Sciences 2004;22(7): 637-643. doi: 10.1080/02640410310001655741

11. Paton CD, Hopkins WG, Vollebregt L. Little effect of caffeine ingestion on repeated sprints in team-sport athletes. Medicine \& Science in Sports \& Exercise 2001;33(5):822 825. doi: 10.1097/00005768-200105000-00023
12. Kedia AW, Hofheins JE, Habowski SM, Ferrando AA, M. Gothard D, Lopez HL . Effects of a pre-workout supplement on lean mass, muscular performance, subjective workout experience and biomarkers of safety. Int $\mathrm{J}$ Med Sci 2014;11(2):116-26. doi: 10.7150/ijms.7073

13. Schneiker KT, Bishop D, Dawson B, Hackett LP. Effects of caffeine on prolonged intermittent-sprint ability in team-sport athletes. Medicine \& Science in Sports Exercise 2006;38(3): 578-585. doi: 10.1249/01.mss.0000188449.18968.62

14. Spencer M, Bishop D, Dawson B, Goodman C. Physiological and metabolic responses of repeated-sprint activities:specific to field-based team sports. Sports Medicine 2005;35(12): 1025-1044.

15. Meeusen R, Roelands B, Spriet LL. Caffeine, exercise and the brain. Nestle Nutr Inst Workshop Ser 2013;76(4):1-12. doi: $10.1159 / 000350223$

16. Jones G. Caffeine and other sympathomimetic stimulants: modes of action and effects on sports performance. Essays Biochem 2008;44:109-23. doi: 10.1042/BSE0440109

17. Davis JK, Green JM. Caffeine and anaerobic performance: ergogenic value and mechanisms of action. Sports Med 2009;39(10):813-32. doi: 10.2165/11317770-000000000-00000

18. Da Silva CG, Cavazzotto TG, Queiroga MR. Suplementação de cafeína e indicadores de potência anaeróbia. Rev Educ Física/UEM 2014;25(1):117-125. doi: 10.4025/reveducfis.v25i1.19306

19. Machado MV, Batista AR, Altimari LR, Fontes EB, Triana RO, Okano AH, Marques AC, Andries Júnior O, Moraes AC. Efeito da ingestão de cafeína sobre os parâmetros da potência crítica. Rev Bras Cineantropom Desempenho Hum. 2010;12(5):49-54. doi: 10.5007/1980-0037.2010v12n1p49

20. Hodgson $A B$, Randell RK, Jeukendrup AE. The metabolic and performance effects of caffeine compared to coffee during endurance exercise. PLoS One 2013;8(4):595-61. doi: 10.1371/journal.pone.0059561 